\title{
Micropropagation and conservation of selected endangered anticancer medicinal plants from the Western Ghats of India
}

\begin{abstract}
Globally, cancer is a constant battle which severely affects the human population. The major limitations of the anticancer drugs are the deleterious side effects on the quality of life. Plants play a vital role in curing many diseases with minimal or no side effects. Phytocompounds derived from various medicinal plants serve as the best source of drugs to treat cancer. The global demand for phytomedicines is mostly reached by the medicinal herbs from the tropical nations of the world even though many plant species are threatened with extinction. India is one of the mega diverse countries of the world due to its ecological habitats, latitudinal variation, and diverse climatic range. Western Ghats of India is one of the most important depositories of endemic herbs. It is found along the stretch of south western part of India and constitutes rain forest with more than 4000 diverse medicinal plant species. In recent times, many of these therapeutically valued herbs have become endangered and are being included under the red-listed plant category in this region. Due to a sharp rise in the demand for plantbased products, this rich collection is diminishing at an alarming rate that eventually triggered dangerous to biodiversity. Thus, conservation of the endangered medicinal plants has become a matter of importance. The conservation by using only in situ approaches may not be sufficient enough to safeguard such a huge bio-resource of endangered medicinal plants. Hence, the use of biotechnological methods would be vital to complement the ex vitro protection programs and help to reestablish endangered plant species. In this backdrop, the key tools of biotechnology that could assist plant conservation were developed in terms of in vitro regeneration, seed banking, DNA storage, pollen storage, germplasm storage, gene bank (field gene banking), tissue bank, and cryopreservation. In this chapter, an attempt has been made to critically review major endangered medicinal plants that possess anticancer compounds and their conservation aspects by integrating various biotechnological tools
\end{abstract}

